\title{
Dynamic Network Slicing for Flexible Radio Access in Tactile Internet
}

\author{
Alireza Shams Shafigh ${ }^{\dagger}$, Savo Glisic ${ }^{\dagger}$ and Beatriz Lorenzo ${ }^{\ddagger}$ \\ $\dagger$ Centre for Wireless Communications, University of Oulu, Finland \\ Email: \{firstname.lastname\}@oulu.fi \\ $\ddagger$ Dept. Telematics, University of Vigo, Spain \\ Email: blorenzo@gti.uvigo.es
}

\begin{abstract}
Tactile Internet (TI) will generate a variety of 5G-enabled use cases with different requirements for latency, throughput and reliability. In this paper, we propose a flexible cloud-based radio access network (FRAN) for TI, where traffic of user equipments (UEs) can be temporary offloaded from the operator-provided networks to user-provided networks if needed. Such a concept enables dynamic network slicing (DNS) where the network architecture is temporally augmented with slices of infrastructure borrowed from user provided network. FRAN is able to support Tactile applications without any basic change to the hardware/software infrastructure in the network. We model DNS system as a two-layer/slice traffic-aware resource allocation framework, where every layer uses a separate two-step matching game in order to serve Tactile users (TUs) and low-priority users (LUs). We propose a subgame Nash stable and two-sided exchange stable concepts as solutions of the proposed two-layer traffic-aware resource allocation. Our numerical results show that network operators and UEs (either TUs or LUs) significantly benefit from deploying the FRAN rather than conventional cloudbased radio access networks (CRANs).
\end{abstract}

Keywords. Tactile Internet, dynamic network architecture, cloud-based radio access network, two-sided exchange stable, dynamic network slicing.

\section{INTRODUCTION}

Nowadays, wireless communication networks are largely focused towards latency tolerant traffic with little attention to delay-sensitive traffic e.g. Tactile Internet applications [1], [2]. In these systems, including Internet of things (IoT) applications, vehicular networks, smart grid, distributed robotics, and other cyber-physical systems, the requirements for latency could be two or three orders of magnitude more stringent than in traditional applications. Tactile Internet will provide a true paradigm shift from content-delivery to skill-set delivery networks, and thereby revolutionize almost every segment of the society [3], [4], [5], [6], [7].

In order to facilitate the emergence of such ultra lowlatency and high-reliability applications, this paper develops a novel cloud-based radio access network (CRAN) system [8], [9] as a new cloud architecture for future mobile network operators (MNOs). This flexible cloud radio network (FRAN) for Tactile Internet (TI) adaptively uses user-provided networks [10] to provide an efficient framework for ultra low-latency communications. In user-provided networks, users share their connectivity and act as access points for other users and so increase the network capacity. DNS is capable to instantly offload current low-priority users (LUs) to userprovided networks and reassign resources to high priority Tactile users (TUs). Incentive and pricing mechanisms have attracted intensive research to motivate users to share their connectivity and establish a dynamic network architecture (DNA) [10], [11], [12], [13], [14]. Here, we further extend DNA concept to make the access network more flexible and self-adjustable to traffic density variations.

The main contributions of our paper are as follows:

- A flexible radio access network (FRAN), based on DNS, optimized for Tactile Internet by combining DNA and CRAN concepts.

- A two-step matching game for traffic-aware resource allocation in FRAN with DNS.

- Load balancing and offloading techniques to support flexibility concept of FRAN.

- Two-sided exchange-stable and Nash stability concepts as solutions of the resource allocation in FRAN.

The rest of the paper is organized as follows. Section II presents the system model. In Section III, the two-layer trafficaware resource allocation problem is presented while Section IV presents the numerical results. Finally, Section V concludes the paper.

\section{SySTEM MOdEL}

We consider a flexible cloud radio access network (FRAN) system based on DNS for Tactile Internet (TI) with $B$ remote radio heads (RRHs) denoted by the set $\mathcal{B}$, which are used to serve $I$ user equipments (UEs) denoted by the set $\mathcal{I}$. We indicate by $\mathcal{K}$ the set of available physical resource blocks (PRBs), which are assigned by the baseband unit (BBU) to $B$ RRHs in the system shown in Fig.1. Furthermore, there are $J$ user-provided access points strengthening the proposed system by offering available resources to their vicinity low-priority users (LUs). The user-provided dynamic access points (DAPs) $\mathcal{J}$ are user devices motivated by operators to share their unused resources. Such dynamic network architecture (DNA) 


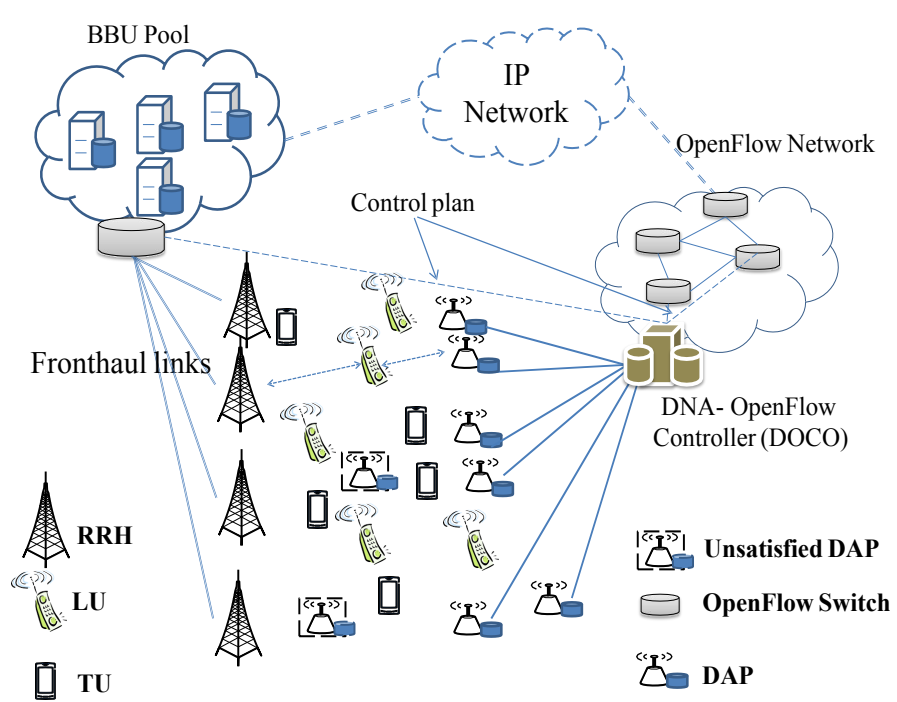

Fig. 1. System Model.

[10] creates an efficient mechanism for providing multiconnectivity for network users. The DNA offloads mobile data from FRAN in order that the operator-provided resources e.g, BBUs and RRHs are used for the Tactile users (TUs).

In the proposed model, there exists two different types of UEs with different requirements: low-priority users (LUs) and high-priority Tactile users (TUs). The former group $\mathcal{I}^{L} \subset \mathcal{I}$ is only concerned about data rate and can be offloaded to DNA networks (user-provided connectivity) if needed. The later group $\mathcal{I}^{T} \subset \mathcal{I}$ is very sensitive to the latency. It needs to be served by FRAN system due to the security and reliability issues. The TUs need specific low-latency communications provided by the FRAN system. LUs, which are delay tolerant, can be temporary offloaded to DNA to make free resources for TUs if FRAN's resources are fully occupied. Every LU $i \in \mathcal{I}^{L}$ requires a minimum data rate $\Gamma_{i}$ to be served by user/operator-provided networks while every $\mathrm{TU} i \in \mathcal{I}^{L}$ can not tolerate latency longer than $\tau_{i}^{t h}$.

Here, we denote by $\mathcal{R}=\{r \mid r \in \mathcal{J} \cup \mathcal{B}\}$ the set of both RRHs and DAPs. Fig.1 presents the FRAN system based on DNA, where operators can extend their access network with minor changes in the current software and hardware infrastructure. The DNA networking paradigm is inherently capable to improve the FRAN system performance in terms of energy efficiency, data rate and delay by serving some of the UEs that have flexible requirements. FRAN offloads delay tolerant users to DNA to provide required QoS for Tactile users. DNA networks charge the network operator (NO) according to the provided resources for LUs. Thus, the NO should deploy an efficient resource allocation approach in such a way that its profit is maximized and UEs requirements are satisfied. In addition to the offloading mechanism in the access network, we use a load balancing technique in the fronthaul network to assign RRHs to BBUs according to users' requirements.

\section{A. Software-Defined Network (SDN) integrates DNA and DNS as a FRAN System}

OpenFlow (OF) standard for software-defined networking defines the communication protocol enabling SDN controllers to directly interact with the forwarding plane of network devices such as switches and routers, so it can efficiently adapt to DNA requirements. A DNA-OpenFlow controller (DOCO) in FRAN is originally responsible for all signaling required in control plan for pricing, reimbursement and offloading. DOCO updates the lists of available DAPs and UEs that want to participate in the offloading process according to the offered incentive $\beta$. It is able to remotely control the DNA networks so that the user-provided slices are included in the network if needed. The proposed FRAN system uses dynamic network slicing concept to support requirements of TUs [15]. The concept is based on the same rational as dynamic spectrum management over conventional fixed allocation of the spectrum. To leverage network slicing properly, the individual segments (radio access network (RAN), radio air interface, RRHs, DAPs and BBUs), which were formerly treated separately, must be examined as a whole. The proposed dynamic network slicing concept extends the conventional slicing vision [7] and addresses properly requirements of TUs. It partitions CRAN and DNA network infrastructures into multiple, logical, end-to-end, virtual network layers or slices . The slices are dynamic and can be adjusted according to the requirements of users in each layer. To provide ultra-reliable connections, more than one slice can be allocated to the TUs. Furthermore, the slices can be locally optimized in order to instantly be adapted to the network changes.

\section{B. TUs' and LUs' preferences}

We assume that every UE has different preferences including throughput and latency. To meet UEs' requirements at least two kinds of slices are defined as follow:

1) High-Throughput Slice (HTS): LUs prefer to be assigned to the nearest access point either RRH or DAP to achieve the maximum possible throughput with power efficiency. Therefore, they can rank available DAP and RRHs in order to create a dynamic slice according to the provided throughput. For every UE $i$ and AP $r \in \mathcal{J} \cup \mathcal{B}$ belonging to a slice, capacity of link $(i, r)$ is calculated as

$$
C_{i, r}=\sum_{s \in \mathcal{S}} \omega_{s} \log \left(1+\frac{P_{i} D_{i, r}^{-\alpha}}{N_{0}+\gamma_{r}(\eta, \mu)}\right), \quad \sum_{r \in \mathcal{J} \cup \mathcal{B}} C_{i, r} \geq \Gamma_{i}^{t h},
$$

where $\omega_{s}, P_{i}, D_{i, r}$ and $N_{0}$ represent the bandwidth of subcarrier $s$, transmit power of UE $i$, distance between $(i, r)$ and the noise respectively. In the above, $\gamma_{r}(\eta, \mu)$ represents the observed interference by $r$ from the set of simultaneous communications activated by matching functions $\eta$ and $\mu$. These functions define the optimum selection of access points by matching algorithms defined in the next section. We will show, in the next section, that $\eta$ and $\mu$ include communications between UEs and RRHs\&DAPs in access and fronthaul networks. In (1), $\mathcal{S}$ denotes the set of available sub-carriers. 
The received signal strength is proportional to $D_{i, r}^{-\alpha}$, where $\alpha$ is the pathloss exponent.

2) Low-Latency Slice (LLS): Every UE $i \in \mathcal{I}$ belonging to LLS, experiences delay $\tau_{i}$ defined as

$$
\tau_{i}=\tau_{i, b}^{A}+\tau_{b, k}^{F}, \quad b \in \mathcal{B}, k \in \mathcal{K},
$$

where $\tau_{i, b}^{A}$ and $\tau_{b, k}^{F}$ represent access and fronthaul delays of UE $i$ served by RRH $b$ and BBU $k$ in the access and fronthaul networks respectively. Here, we assume that every UE $i$ has an arrival rate $\lambda_{i}$. Furthermore, propagation delay of fronthaul due to using high speed communication links is negligible. Thus, the access delay of every UE $i$ associated with RRH $b$ can be calculated as

$$
\tau_{i, b}^{A}=\frac{1}{\kappa_{b}-\lambda_{i}}, \quad \kappa_{b}>\lambda_{i}
$$

where $\kappa_{b}$ represents service rate of RRH $b$. In the BBU pool, every RRH is associated at most to one BBU which can serve more than one RRH (many-to-one association). Therefore, delay of a given RRH $b$ that is served by a BBU $k$ can be calculated as

$$
\tau_{b, k}^{F}=\frac{1}{\kappa_{k}-\lambda_{b}-\sum_{b^{\prime} \in \mathcal{B}_{k} \backslash b} \lambda_{b^{\prime}}}, \quad \kappa_{k}>\left(\lambda_{b}+\sum_{b^{\prime} \in \mathcal{B}_{k} \backslash b} \lambda_{b^{\prime}}\right)
$$

where $\kappa_{k}$ and $\lambda_{b}$ represent the service rate of $k \in \mathcal{K}$ and arrival rate of $b \in \mathcal{B}$ respectively. In the above, $\mathcal{B}_{k}$ is the subset of RRHs assigned to BBU $k$. In the proposed trafficaware resource allocation process, if current resources are not enough for providing required QoS of TUs, some LUs would be offloaded to DNA networks. Furthermore, the fronthaul network can be adapted by load balancing to avoid allocating overloaded BBUs to the incoming TUs.

\section{Utility and Profit Functions}

Future network operators with Tactile services would be able to provide ultra low-latency communications for UEs. Based on the network condition, LUs might be offloaded to DNA networks by offering some incentives. This offloading process imposes extra cost to the operator. For every offloaded UE, a new network slice is created over DNA. Therefore, profit function of NO, which includes cost of offloading and gains from TUs and LUs, is given as

$$
\Pi=\sum_{i \in \mathcal{I}^{T}} \pi_{i}^{T}+\sum_{i \in \mathcal{I}^{L}} \pi_{i}^{L}-\vartheta \times\left|\mathcal{I}_{D N A}^{L}\right|,
$$

where the first and second terms represent the benefit of the NO from serving TUs and LUs respectively. The offloading procedure imposes the cost $\vartheta \times\left|\mathcal{I}_{D N A}^{L}\right|$ to the operator, where $\vartheta$ and $\mathcal{I}_{D N A}^{L} \subset \mathcal{I}$ are the offloading cost and the list of accepted LUs for offloading by DNA network respectively. Therefore, every network operator should decide about the number of LUs to be offloaded to DNA networks in order to meet the requirements of TUs.

In UE's side, we model satisfaction of TUs with a utility function given as

$$
\pi_{i}^{T}=C_{i}-\left[\tau_{i}-\tau_{i}^{t h}\right]^{+} \zeta\left(\tau_{i}\right)
$$

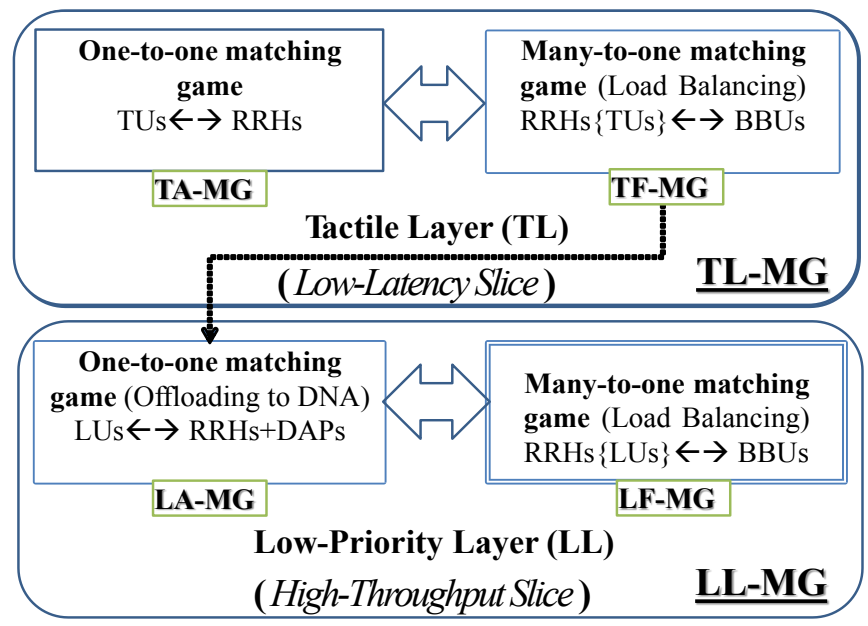

Fig. 2. Two-layer traffic-aware resource allocation in FRAN.

where $C_{i}=\sum_{b \in \mathcal{B}} C_{i, b}$ represents the achieved capacity of TU $i$. The penalty function $\zeta\left(\tau_{i}\right)$ reflects the delay sensitivity of TUs. If $\tau_{i}>\tau_{i}^{t h}, \zeta\left(\tau_{i}\right) \rightarrow+\infty$. In (6), operand [ ] ${ }^{+}$returns 0 if $\tau_{i}<=\tau_{i}^{t h}$, otherwise 1 . So, if $\tau_{i}>\tau_{i}^{t h}$ the penalty function $\zeta\left(\tau_{i}\right)$ imposes a huge amount of penalty value to the operator.

LUs are only concerned about data rate and their utility function can be denoted as

$$
\pi_{i}^{L}=C_{i}, \quad C_{i} \geq \Gamma_{i}^{t h} .
$$

Existing pricing and incentives mechanisms in DNA [10], [16], [17] can be easily extended to motivate access points to cooperate in FRAN to build up an extra slice in the network.

\section{TWO-LAYER TRAFFIC-AWARE RESOURCE ALLOCATION IN FRAN}

We develop a framework to assign user and operatorprovided resources (e.g. link capacity) to TUs and LUs in such a way that all players (network operators and UEs) meet their requirements. Network operator optimizes resource utilization ratio while UEs have their own quality of service requirements. Here, the proposed traffic-aware resource allocation (See Fig.2) consists of two different layers for TUs and LUs. In the first layer (high priority slice), TUs are assigned to RRHs and RRHs to BBUs in access and fronthaul networks respectively. In the second layer (low priority layer), LUs are associated to DAPs\&RRHs and RRHs to BBUs through offloading and load balancing techniques in access and fronthaul networks respectively. If the delay of a typical TU is higher than its requirement, load balancing technique is used to rearrange the allocation scheme in fronthaul network. If it fails, active LUs are blocked (offloaded from the FRAN to DNA) to reduce the experienced-delay by the TUs.

In the proposed model, the efficiency of Tactile layer (TL) significantly depends on low-priority-layer (LL) which is a function of the pricing and incentive mechanisms of operator. Effective pricing and incentive mechanisms can motivate DAPs to participate in the resource sharing process. According 
to Fig.1, unsatisfied DAPs do not participate in offloading mechanism.

\section{A. Centralized Matching Game Model for Each Layer in FRAN}

We develop two separate two-step matching games [18] in response to TI request for low-latency communications (Low-Latency Slice) that requires to be served immediately (See Fig.3) and tolerant LUs request for high-throughput communications (High-Througput Slice), which can be also provided by DNA networks (See Fig.4). In the proposed game, shown in Fig.2, there are four different matching games. For the access networks of TL and LL layers, two one-toone sub-matching games TA-MG and LA-MG are defined between (TUs, RRHs) and (LUs, DAPs\&RRHs) respectively. In the fronthaul network, two many-to-one matching games are defined between (RRHs, BBUs) in both layers. The only difference in the proposed many-to-one matching games is the type of UEs being served. We call many-to-one matching games in fronthaul network of TL and LL layers as TFMG and LF-MG respectively. Therefore, Tactile and highthroughput slices consist of (TA-MG, TF-MG) and (LA-MG, LF-MG) matching games respectively.

Definition 1. A high-priority Tactile Layer (low-latency slice) matching game (TL-MG) is a matching function $\eta$ defined over $\mathcal{I}^{T} \times \mathcal{B} \times \mathcal{K}$ such that $|\eta(i)|=1,|\eta(k)|=r_{k}$ and $|\eta(b)|=1$, where $\eta(i)=\{(b, k) \in \mathcal{B} \times \mathcal{K} \mid(i, b, k) \in \eta\}, \eta(b)=\{(i, k) \in$ $\left.\mathcal{I}^{T} \times \mathcal{K} \mid(i, b, k) \in \eta\right\}, \eta(k)=\left\{(i, b) \in \mathcal{I}^{T} \times \mathcal{B} \mid(i, b, k) \in \eta\right\}$ and $r_{k}$ is the number of RRHs assigned to BBU $k$.

Thus, outcome of TL-MG is a matching function $\eta$ providing the best possible low-latency communications (LLCs) for TUs such that $\forall i \in \mathcal{I}^{T}, \tau_{i}<=\tau_{i}^{t h}$ and $C_{i} \geq \Gamma_{i}^{t h}$. According to the proposed framework, TUs are served first by FRAN (operator-provided resources) and then LUs are decided to associated with either DNA or FRAN without harming TUs. TL-MG uses a load balancing mechanism in the fronthaul network to optimize the performance of TUs.

The LL layer (high-throughput slice) is used to enhance resource allocation efficiency by offloading and load balancing techniques. The general low-priority layer matching game shown in Fig. 4 is defined as

Definition 2. A low-priority layer matching game (LL-MG) is a matching function $\mu[\eta]$ defined over $\mathcal{I}^{L} \times \mathcal{R}[\eta] \times \mathcal{K}[\eta]$ such that $|\mu(i)|=1,|\mu(k)|=r_{k}$ and $|\mu(r)|=1$, where $\mu(i)=\{(r, k) \in \mathcal{R}[\eta] \times \mathcal{K}[\eta] \mid(i, r, k) \in \mu\}, \mu(r)=\{(i, k) \in$ $\left.\mathcal{I}^{L} \times \mathcal{K}[\eta] \mid(i, r, k) \in \mu\right\}, \mu(k)=\left\{(i, r) \in \mathcal{I}^{L} \times \mathcal{R}[\eta] \mid(i, r, k) \in\right.$ $\mu\}, r_{k}$ is the number of RRHs assigned to BBU $k$ and operand [.] denotes the current matching function of TL-MG.

Thus, still under LL-MG, $\tau_{i}<=\tau_{i}^{t h}$ and $C_{i} \geq \Gamma_{i}^{t h}$, $\forall i \in \mathcal{I}$.The outcomes of TL-MG and LL-MG are matching functions $\eta$ and $\mu$ for jointly associating (TUs, LUs) to (RRHs, RRHs\&DAPS) and RRHs to BBUs in different slices (See Fig.2).

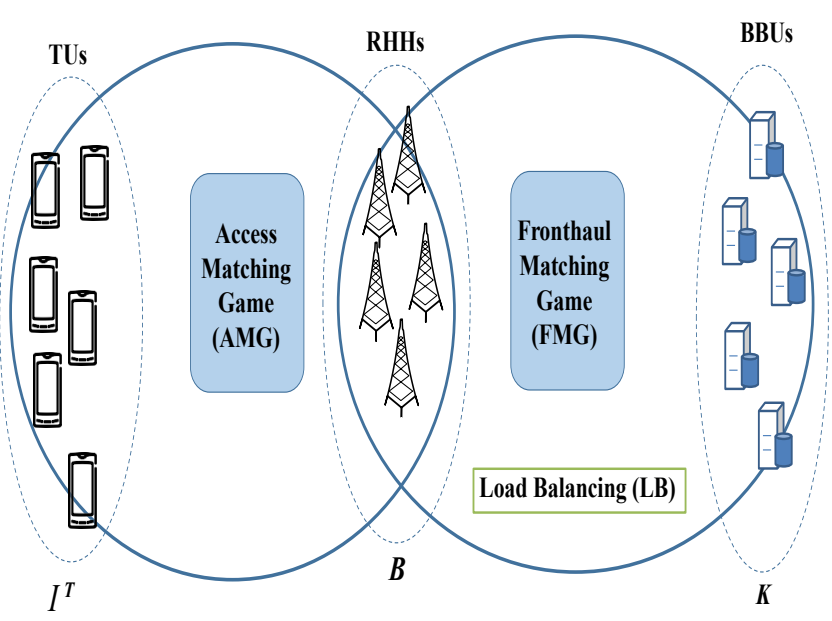

Fig. 3. Matching Model in hi-priority Tactile layer (TL-MG).

According to the proposed network slicing model, the preferences of a player depend on other players' choices having an impact on cochannel interference in the access network and delay in the fronthaul network. These externalities may degrade the performance of players especially TUs which need very low-latency communications. These externalities should be addressed in the sub-games in order to reach a stable matching as a solution of both TL-MG and LL-MG games. To deal with this challenge we look at a new stability concept based on the idea of swap-matching [19]. In a swap-matching, the set of players that benefit from changing their current matching is called a blocking set.

Definition 3. $\mathcal{S}_{u}$ is a blocking set of current matching if and only if there exist a pair of UEs belonging to $\mathcal{S}_{u}$ that prefer to switch their DAPS/RRHs while keeping all other UEs' associations the same. For RRHs, $\mathcal{S}_{r}$ is a blocking set of RRHs if and only if there exist a pair of RRHs belonging to $\mathcal{S}_{r}$ that prefer to switch their BBUs while keeping all other RRHs' associations the same.

According to the definition of blocking sets, in the access and fronthaul matching, a matching is stable if does not exist any blocking set. Here, we need a new stability concept with assumption that all players in a blocking set must approve the swap matching. A BBU serving a critical TU may not accept another user if accepting the new user degrades the performance of the TU. Therefore, we introduce a new stability concept [19] as two-sided exchange-stable (TSES) matching defined as

Theorem 1. A matching $\psi \in\{\eta, \mu\}$ is two-sided exchangestable (TSES) if and only if there is not any pair of either UEs $\left(i, i^{\prime}\right), \forall i, i^{\prime} \in \mathcal{I}$ or pair of RRHs $\left(r, r^{\prime}\right), \forall r, r^{\prime} \in \mathcal{R}$ such that in access/fronthaul matching game $(A M G / F M G)$ :

$$
\begin{aligned}
\text { (a) } \forall q \in\left\{x, x^{\prime}, \psi(x), \psi^{\prime}\left(x^{\prime}\right)\right\} & \pi_{q}\left(\psi^{\prime}\right) \geq \pi_{q}(\psi), \text { and } \\
\text { (b) } \exists q \in\left\{x, x^{\prime}, \psi(x), \psi^{\prime}\left(x^{\prime}\right)\right\} & \pi_{q}\left(\psi^{\prime}\right)>\pi_{q}(\psi), \\
\forall x \in\{i, r\}, x^{\prime} \in\left\{i^{\prime}, r^{\prime}\right\}, &
\end{aligned}
$$

where $\psi^{\prime}$ is a typical matching function. 


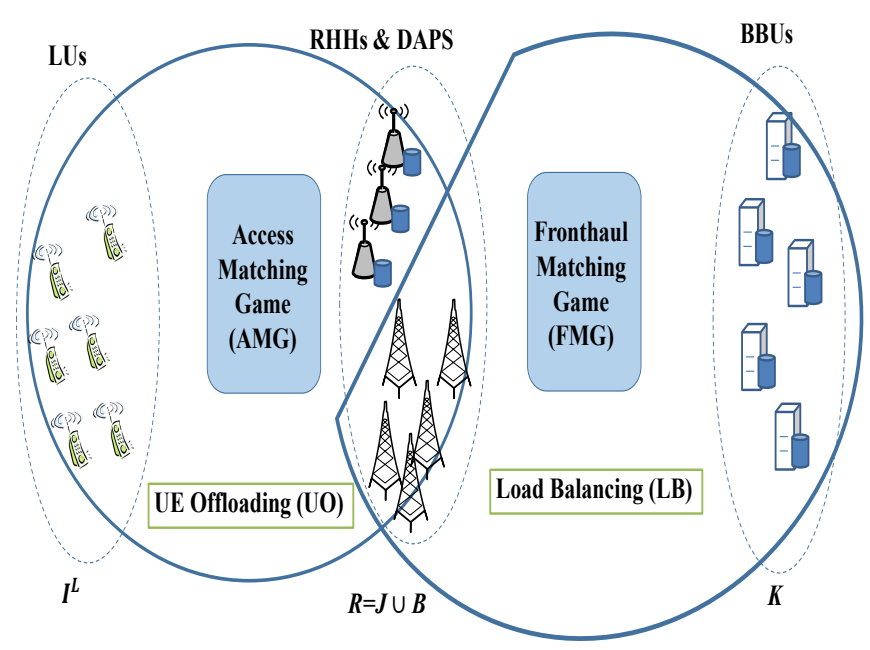

Fig. 4. Matching Model in low-priority layer (LL-MG).

Proof. As mentioned before, existence of at least one blocking set can harm the stability of a matching game. According to the conditions (a) and (b) in (8), all blocking members approve the swapping operation if only if they do not loose any profit, otherwise the set can not reach the stability.

\section{B. Decomposing Centralized Matching Games into Two-Sided Exchange-Stable Matching (TSES)}

Solving the matching games TL-MG and LL-MG in their original form is complicated and impractical. Here, we are looking for a distributed resource allocation algorithm for self-organizing and autonomous players. We decompose the original matching games TL-MG and LL-MG into access and fronthaul sub-matching games, AMG and FMG respectively. Then, we use an iterative algorithm to find TSES matching in these sub-games (See Fig.2).

Due to the requirements of TUs, their resource allocation process is independent from LUs. Thus, we propose two different resource allocation algorithms for serving TUs and LUs. However their resource allocation procedure is almost the same especially in the FMG, where RRHs are assigned to BBUs. The main difference between these two mechanisms in TL and LL layers is that DAPs are excluded from the access network in TL. It is reasonable because the network should provide strict constraints for TUs. Every DAP $j$ has its own Internet connection, processing and storage capacities but its intermittent availability is unsuitable to guarantee any QoS level for Tactile users.

In order to find a stable matching for TL-MG/LL-MG, we can use the following Theorem.

Theorem 2. A matching result of (TL-MG, LL-MG) is TSES if their sub-matching games (TA-MG, LA-MG) in the access and (TF-MG, LF-MG) in fronthaul networks are TSES respectively.

Proof. The proof is straightforward. If one of the sub-games is not in its TSES state, it means that there is at least one blocking set in the matching.
Due to the rationality of players and their strict requirements, we define a Nash stability concept for TL-MG and LLMG games by introducing a sub-Nash stable (SNS) concept for sub-games. Nash stable provides a suitable framework for players with strict requirements in the proposed traffic-aware resource allocation mechanism.

1) Sub-game Nash stability for AMGs: We define preference relations $\succ_{i}$ and $\succ_{b}$ over the sets of $\mathcal{B}$ and $\mathcal{I}$ so that players in $\mathcal{I}$ and $\mathcal{B}$ are able to rank each other respectively.

Lemma 1. A strategy $\eta_{A}^{*}$ for $T A-M G$ is called a SNS, if $\forall i, i^{\prime} \in$ $\mathcal{I}^{T}$ and $\forall b, b^{\prime} \in \mathcal{B}$, there is not any player in $\left\{i, i^{\prime}, b, b^{\prime}\right\}$ who can benefit from moving current matching $\eta_{A}^{*}$ to another matching $\eta_{A}$. In other words, the following condition always hold $\forall \eta_{A} \in \overline{\eta_{A}}$ :

$$
\begin{aligned}
& \text { (a) }\left(i / b, \eta_{A}^{*}\right) \underset{b / i}{\succ}\left(i^{\prime} / b^{\prime}, \eta_{A}\right) \leftrightarrow \pi_{b / i}\left(\eta_{A}^{*}\right) \geq \pi_{b / i}\left(\eta_{A}\right), \\
& \text { (b) }\left(i / b, \eta_{A}^{*}\right) \underset{b / i}{\succ}\left(i^{\prime} / b^{\prime}, \eta_{A}\right) \leftrightarrow \pi_{b / i}\left(\eta_{A}^{*}\right)>\pi_{b / i}\left(\eta_{A}\right),
\end{aligned}
$$

where $\overline{\eta_{A}}$ is the set of possible TSES matching for TA-MG.

Proof. The first condition implies that player $b$ or $i$ prefers $\eta_{A}^{*}$ to $\eta_{A} \in \overline{\eta_{A}}$ since it gains benefit or at least its utility remains the same in the worse case scenario. The second condition denotes that at least one of the players in $\left\{i, i^{\prime}, b, b^{\prime}\right\}$ benefit from $\eta_{A}^{*}$. So, each condition assures that $\eta_{A}^{*}$ is a SNS, otherwise at least one of the condition does not hold.

For LA-MG which is a matching game for offloading technique, we have the following Lemma for SNS:

Lemma 2. A strategy $\mu_{A}^{*}$ for $L A-M G$ is called a $S N S$, if $\forall i, i^{\prime} \in \mathcal{I}^{L}, \forall r, r^{\prime} \in \mathcal{R}$, there is not any player in $\left\{i, i^{\prime}, r, r^{\prime}\right\}$ who can benefit from moving his current matching $\mu_{A}^{*}$ to another matching $\mu_{A}$. In other words, the following condition always hold $\forall \mu_{A} \in \overline{\mu_{A}}$ :

$$
\begin{aligned}
& \text { (a) } \quad\left(i / r, \mu_{A}^{*}\right) \underset{r / i}{\succ}\left(i^{\prime} / r^{\prime}, \mu_{A}\right) \leftrightarrow \pi_{r / i}\left(\mu_{A}^{*}\right) \geq \pi_{r / i}\left(\mu_{A}\right), \\
& \text { (b) } \quad\left(i / r, \mu_{A}^{*}\right) \underset{r / i}{\succ}\left(i^{\prime} / r^{\prime}, \mu_{A}\right) \leftrightarrow \pi_{r / i}\left(\mu_{A}^{*}\right)>\pi_{r / i}\left(\mu_{A}\right),
\end{aligned}
$$

where $\overline{\mu_{A}}$ is the set of possible TSES matching for LA-MG.

Proof. Proof is straightforward.

The above Lemma implies that LU are offloaded according to the current matching function of TA-MG.

2) Sub-game Nash Stability for FMGs: We define a preference relation $\underset{k}{\succ}$ over the set of $\mathcal{B}$ so that players (BBUs) are able to rank ${ }^{k}$ RRHs. Here, we define load balancing submatching games (TF-MG and LF-MG) in both layers as:

Lemma 3. A strategy $\left(\mu_{F}^{*} / \eta_{F}^{*}\right)$ is called a SNS for $L F-M G / T F-M G, \forall k, k^{\prime} \in \mathcal{K}$ and $\forall b, b^{\prime} \in \mathcal{B}$, if there is not any player in $\left\{b, b^{\prime}, k, k^{\prime}\right\}$ who can benefit from moving his current matching $\mu_{F}^{*} / \eta_{F}^{*}$ to another matching $\mu_{F} / \eta_{F}$.

(a) $\left(b, \mu_{F}^{*} / \eta_{F}^{*}\right) \succ_{k}\left(b^{\prime}, \mu_{F} / \eta_{F}\right) \leftrightarrow \pi_{k}\left(\mu_{F}^{*} / \eta_{F}^{*}\right) \geq \pi_{k}\left(\mu_{F} / \eta_{F}\right)$,

(b) $\left(k, \mu_{F}^{*} / \eta_{F}^{*}\right) \succ_{b}\left(k^{\prime}, \mu_{F} / \eta_{F}^{\prime}\right) \leftrightarrow \pi_{b}\left(\mu_{F}^{*} / \eta_{F}^{*}\right)>\pi_{b}\left(\mu_{F} / \eta_{F}\right)$, 


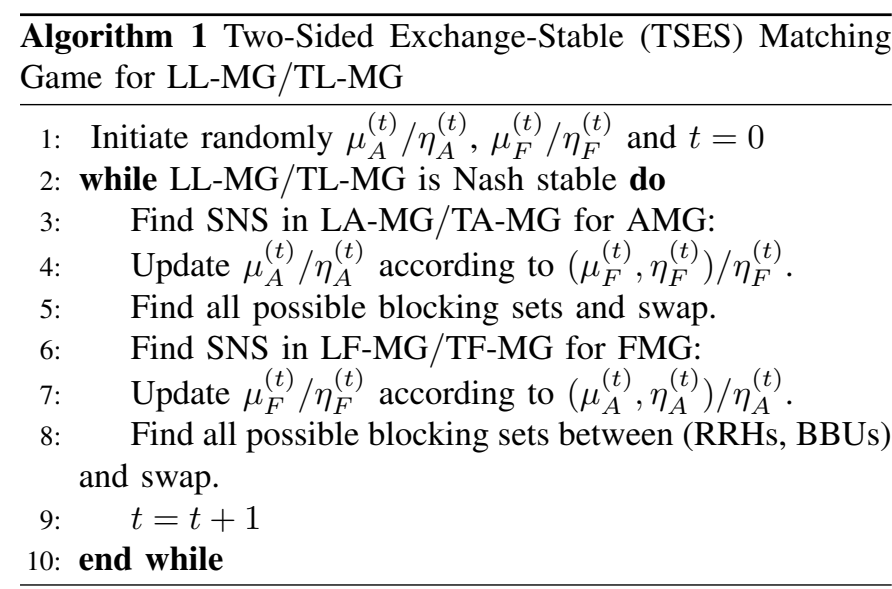

where $\overline{\mu_{F}} / \overline{\eta_{F}}$ is the set of possible TSES matching between RRHs and BBUs in LL/TL layer respectively.

Proof. Proof is straightforward.

We can define Nash stable for the TL-MG/LL-MG by the following theorem:

Theorem 3. A matching result $\left(\mu_{A}^{*}, \mu_{F}^{*}\right) /\left(\eta_{A}^{*}, \eta_{F}^{*}\right)$ of LL-MG/TL-MG is Nash stable if $\mu_{A}^{*} / \eta_{A}^{*}$ and $\mu_{F}^{*} / \eta_{F}^{*}$ are Nash stables for sub-matching games $L A-M G / T L-M G$ and $L F-M G / T L-M G$ respectively.

Proof. If one of the sub-games is not Nash stable, at least one of players can improve its benefit by blocking current matching result. Thus, LF-MG/TL-MG is Nash stable if only if both sub-games are Nash stable.

Thus, we find a Nash stable by iteratively finding SNSs for sub-games. Notice that we use a SNS of one sub-game as an input for the another sub-match game. For example, we use $\mu_{A}^{*}$ in AMG as initial state of FMG and find $\mu_{F}^{*}$, which is used as initial state of AMG in the next iteration. This procedure continues until it converges. We propose Algorithm 1 to find a Nash stable matching for LL-MG/TL-MG. This algorithm consists of two parts, which belong to access matching game (AMG) and fronthaul matching game (FMG). In AMG for LL layer, $\mu_{A}^{(t)}$ is updated based on $\left(\mu_{F}^{(t)}, \eta_{F}^{(t)}\right)$ while $\eta_{A}^{(t)}$ is updated based on $\eta_{F}^{(t)}$ in TL layer. This means that LUs assignment should be done precisely without harming TUs.

First, we run Algorithm 1 for Tactile slices to find Nash stable for Tactile users independent of LUs. Then, we run Algorithm 1 for LUs such that TUs still meet their requirements.

\section{Simulation Results}

In this section, we present numerical results to validate the theoretical analysis of the previous sections and provide some further insights into the operation of two-layer traffic-aware resource allocation model. In the analysis we use random network topology with parameters from Table I.

Fig. 5 presents the utility of UEs versus the number of iterations with the number of TUs and LUs as parameters. According to the achieved results, the proposed algorithm requires at least 10 iterations to reach Nash stability in Tactile
TABLE I

SIMULATION PARAMETERS

\begin{tabular}{|c|c|}
\hline Parameter & Value \\
\hline Simulation Area & $1000^{2}$ \\
\hline$\lambda$ & 10 Packet/sec \\
\hline$I$ & $5-40$ \\
\hline$J$ & $5-20$ \\
\hline$B$ & 5 \\
\hline $\mathrm{K}$ & 10 \\
\hline Transmission power & $30 \mathrm{dBm}$ \\
\hline UEs' and BSs'positions & random \\
\hline
\end{tabular}

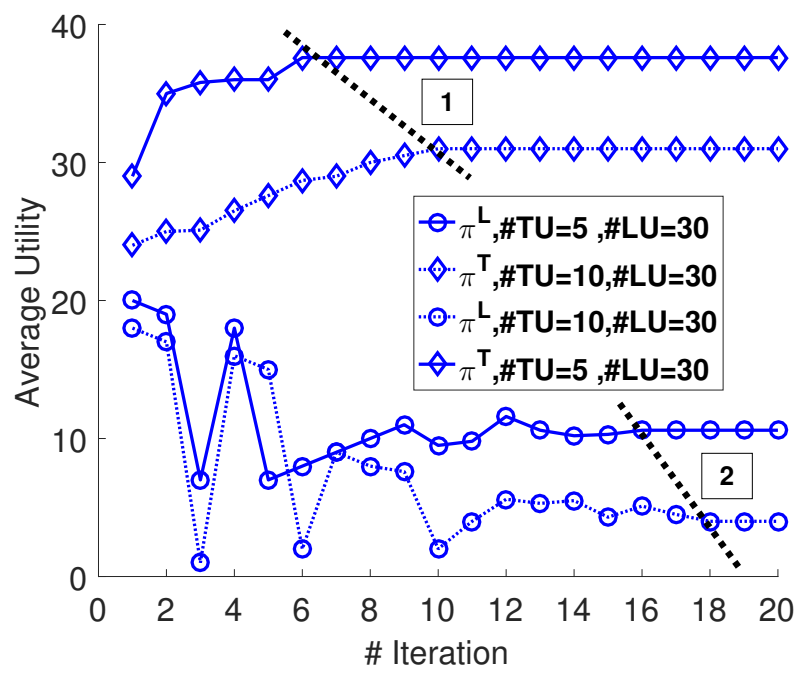

Fig. 5. Average utility versus the number of iterations.

layer (marker 1). Next, LUs use load-balancing and offloading techniques to reach Nash stability in the high-throughput network with about 20 iterations. As expected, increasing of the number of TUs can significantly reduce the average utility of other users.

Fig. 6 shows the average utility versus the number of DAPs with the number of TUs and LUs as parameters. Introducing more DAPs into the FRAN system results in a significant increase in the utility of LUs since DNA can only handle LUs. By increasing the number of DAPs from 2 to 20 the average utility of LUs is improved by factors between 3 and 6 . There is also an improvement in TUs' utility due to the reduction of traffic load by offloading LUs. DNA can enhance TUs' utility at least $15 \%$ by introducing DAPs.

Fig. 7 presents the running time of the proposed algorithm versus the number of LUs with the number of DAPs as parameters. According to the achieved results, the running time of the algorithm is less than $40 \mathrm{~ms}$ for allocating resources to the both layers while the resource allocation for Tactile users takes less than $10 \mathrm{~ms}$ in order to satisfy the requirements of Tactile applications.

\section{CONCLUSION}

This paper proposes a flexible framework networking called FRAN based on using DNA networking paradigm for dynamic network slicing for Tactile Internet applications. In this kind 


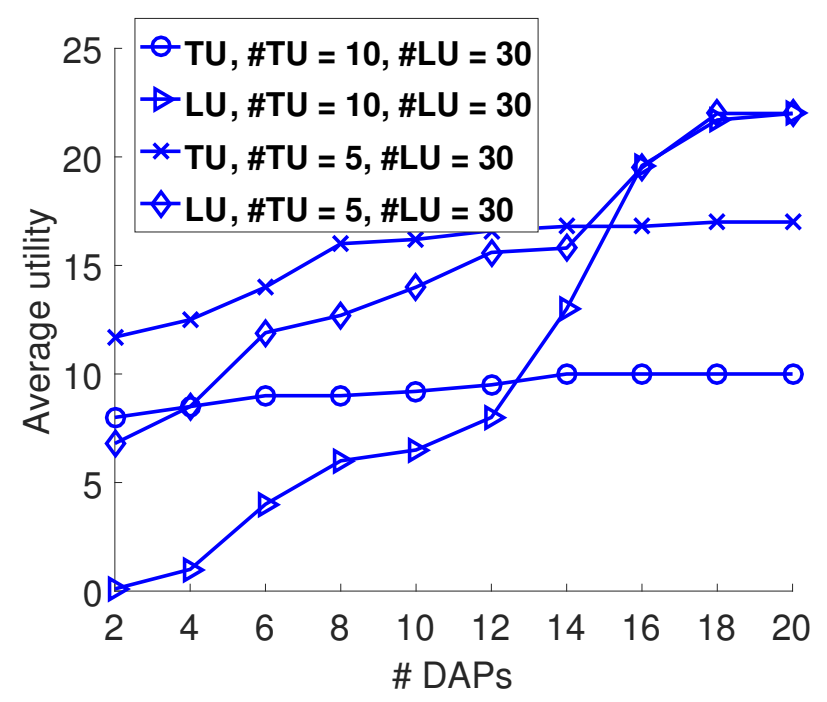

Fig. 6. Average utility versus the number of DAPs.

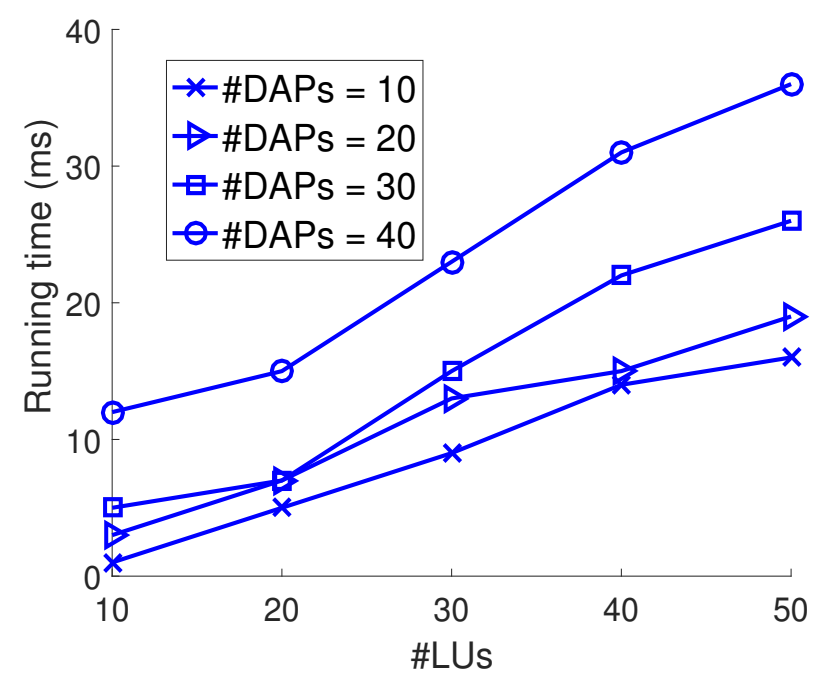

Fig. 7. Running time versus the number of LUs ( TUs =10).

of networking model, operators motivate users to share their resources instead of installing costly hardware/software infrastructure equipment everywhere. A key contribution of our paper is two-step matching game model for the proposed twolayer traffic-aware resource allocation mechanism in which load balancing and offloading techniques are used to improve the performance of FRAN. As a solution for such a model we have developed two-sided exchange-stable and Nash stability concepts. Our numerical results indicate that combining DNA and CRAN is an efficient networking model to extend dynamically current networks for supporting Tactile Internet applications without any extra investment into infrastructure.

As possible extensions of this work, we are planning to consider terminals equipped with multiple antennas and develop beam forming schemes to select the most appropriate APs that satisfy the QoS requirements and maximize throughput.

\section{REFERENCES}

[1] G. P. Fettweis, "The Tactile Internet: applications and challenges," IEEE Vehicular Technology Magazine, vol. 9, no. 1, pp. 64-70, 2014

[2] A. Aijaz, M. Simsek, M. Dohler, and G. Fettweis, "Shaping 5G for the Tactile Internet," 5G Mobile Communications, pp. 677-691, 2017.

[3] ITU, "Standards specifications," http://www.3gpp.org/specifications.

[4] G. Fettweis, "The opportunities of the Tactile Internet-and a challenge for future electronics," in 24th ICFPL (International Conference on Field Programmable Logic and Applications), Munich, Germany, 2014.

[5] E. Dahlman, G. Mildh, S. Parkvall, J. Peisa, J. Sachs et al., "5G wireless access: requirements and realization," IEEE Communications Magazine, vol. 12 , no. 52, pp. 42-47, 2014.

[6] P. et al, "Scenarios requirements and KPIs for $5 \mathrm{G}$ mobile and wireless system-deliveable 1.1," ICT-317669METISproject, 2013.

[7] MGMN, "5G white paper[online] available:," https://www.ngmn.org/ uploads/media/NGMN_5G_White_Paper_V1_0.pdf, 2015.

[8] M. Y. Lyazidi, N. Aitsaadi, and R. Langar, "Dynamic resource allocation for Cloud-RAN in LTE with real-time BBU/RRH assignment," in Communications (ICC), 2016 IEEE International Conference on. IEEE, 2016, pp. 1-6.

[9] J. E. Mitchell, "Integrated wireless backhaul over optical access networks," Journal of Lightwave Technology, vol. 32, no. 20, pp. 3373-3382, 2014.

[10] A. S. Shafigh, B. Lorenzo, S. Glisic, J. Pérez-Romero, L. A. DaSilva, A. B. MacKenzie, and J. Roning, "A framework for dynamic network architecture and topology optimization," IEEE/ACM Transactions on Networking, vol. 24, no. 2, pp. 717-730, 2016.

[11] M. H. Afrasiabi and R. Guérin, "Pricing strategies for user-provided connectivity services." pp. 2766-2770, 2012.

[12] L. Gao, G. Iosifidis, J. Huang, and L. Tassiulas, "Hybrid data pricing for network-assisted user-provided connectivity," Proceedings IEEE INFOCOM, pp. 682-690, 2014

[13] J. Perez-Romero, J. Sanchez-Gonzalez, R. Agusti, B. Lorenzo, and S. Glisic, "Power efficient resource allocation in a heterogeneous network with cellular and D2D capabilities," IEEE Transactions on Vehicular Technology, vol. 65, no. 11, pp. 9272-9286, 2016.

[14] B. Lorenzo, F. J. Gonzalez-Castano, and Y. Fang, "A novel collaborative cognitive dynamic network architecture," IEEE Wireless Communications, vol. 24, no. 1, pp. 74-81, 2017.

[15] NOKIA, "Dynamic end-to-end network slicing for 5G," http://resources.alcatel-lucent.com/asset/200339.

[16] A. S. Shafigh, P. Mertikopoulos, and S. Glisic, "A novel dynamic network architecture model based on stochastic geometry and game theory," IEEE International Conference on Communications (ICC), 2016.

[17] A. S. Shafigh and S. Glisic, "User-centric spectrum sharing in dynamic network architecture," IEEE Globecom Workshops (GC Wkshps), pp. $1-7,2016$.

[18] B. Lorenzo and F. J. Gonzalez-Castano, "A matching game for data trading in operator-supervised user-provided networks," IEEE International Conference on Communications (ICC), pp. 1-7, 2016.

[19] A. C. B. H. E. Bodine-Baron, C. Lee and A. Wierman, "Peer effects and stability in matching markets," In Proc. of Intel Conf. on Algorithmic game theory, pp. 117-129, 2011. 